\title{
Heat shock proteins in chronic kidney disease
}

\author{
Kinga Musial • Danuta Zwolińska
}

Received: 10 September 2010 /Revised: 25 October 2010 /Accepted: 26 October 2010 /Published online: 31 December 2010

(C) The Author(s) 2010. This article is published with open access at Springerlink.com

\begin{abstract}
Heat shock proteins (HSP) form a heterogenous, evolutionarily conserved group of molecules with high sequence homology. They mainly act as intracellular chaperones, protecting the protein structure and folding under stress conditions. The extracellular HSP, released in the course of damage or necrosis, play a pivotal role in the innate and adaptive immune responses. They also take part in many pathological processes. The aim of this review is to update the recent developments in the field of HSP in chronic kidney disease (CKD), in regard to three different aspects. The first is the assessment of the role of HSP, either positive or deleterious, in the pathogenesis of CKD and the possibilities to influence its progression. The second is the impact of dialysis, being a potentially modifiable stressor, on HSP and the attempt to assess the value of these proteins as the biocompatibility markers. The last area is that of kidney transplantation and the potential role of HSP in the induction of the immune tolerance in kidney recipients.
\end{abstract}

Keywords Heat shock proteins . Chronic kidney disease . Hemodialysis $\cdot$ Peritoneal dialysis $\cdot$ Kidney transplantation

\section{Introduction}

Stress response is one of the most evolutionarily conserved reactions to a wide variety of unfavorable physiological or environmental conditions. Heat shock proteins (HSP) constitute a heterogeneous group of main players in this defense mechanism, characteristic for both prokaryotic and

\footnotetext{
K. Musiał • D. Zwolińska $(\bowtie)$

Department of Pediatric Nephrology,

Wrocław Medical University,

M.Skłodowskiej - Curie 50/52,

50-369, Wrocław, Poland

e-mail: zwolin@nefped.am.wroc.pl
}

eukaryotic organisms [1]. A growing body of evidence has expanded the role of HSP, which have long been regarded solely as intracellular chaperones, beyond their cytoprotective function. Current knowledge has given the opportunity to concentrate on the immunomodulatory activity of the extracellular HSP, influencing the innate and adaptive immune responses or various pathologies with the component of the disturbed immunity, such as atherogenesis, cancer, or chronic kidney disease [2].

HSP have been classified, according to their molecular weight, into five groups: small HSP (incl. Hsp27), HSP60, HSP70, HSP90, and HSP110. Among these, HSP60, HSP70, and HSP90 have been studied most extensively due to their engagement in the chaperone activity and a vast role of extracellular forms in the immune response [3]. In each of these groups, the two types of HSP can be found: stress-induced isoforms (Hsp60, Hsp70, Hsp90 $\alpha$ ), as well as those constitutively expressed and independent of the stress conditions (Hsc70, Hsp90ß).

The main goal for the chaperones, discussed widely in recent reviews, is to preserve cell survival by maintaining the vital functions of proteins $[1,3]$. Therefore, HSP regulate the response to any detrimental factors, including temperature, radiation, hypoxia, toxins or infectious agents, by controlling the three-dimensional structure of the newly synthesized proteins, preventing their misfolding or degradation. Stress response may evoke the release of HSP outside the cell, as an effect of an active transport or a cell disintegration due to the infection, damage, or necrosis [2]. Then the extracellular part of the HSP story begins.

\section{The extracellular HSP in the immune system}

Although the presence of the circulating HSP and the antibodies against them has been known for more than a 
decade $[4,5]$, there is still much controversy about the role of the extracellular forms of these proteins. The complexity of the potential HSP impact on the immunity may be the effect of a variety of functions that these proteins have.

The "danger theory" [6] has placed the extracellular HSP in the role of "danger signals"-where the molecules become released only under pathological conditions by the injured or necrotic cells, thus giving an explicit warning signal to the system of the innate immunity. The response to that danger comes from the APC (antigen presenting cells), recognizing HSP through the conserved pattern recognition receptors (PRR), including the Toll-like receptors (TLR). That interaction subsequently triggers APC to produce the proinflammatory cytokines and to activate the nuclear factor $(\mathrm{NF})-\mathrm{kB}$, thus initiating the adaptive immune response and the presentation of antigens to the cytotoxic $\mathrm{T}$ cells [7].

HSP can also stimulate the production of cytokines by monocytes and macrophages, as well as the expression of the adhesion molecules on the endothelial cells. The proinflammatory activity of Hsp60 is certain, whereas the ambiguous function of Hsp70, inducing both the proinflammatory (IL-6, TNF- $\alpha$ ), and anti-inflammatory (IL10) cytokines, may be a matter of speculation. Similarly, Hsp90 in the in vitro investigation has stimulated IFN- $\gamma$ and IL-4 production, activating both Th1 and Th2 lymphocytes [8]. Large clinical studies also gave contradictory results of either protective $[9,10]$ or deleterious [11] effects of Hsp70 in the acute coronary syndrome.

HSP are also known as physiological adjuvants, strengthening the immune response by forming the complexes with antigens, also carcinogens, ready to be transformed by APC [for review see 12].

Last, but not least, there exists a theory of molecular mimicry [13, 14]. The high HSP homology among the species may lead to the dysfunctional immune response. In detail, the similarity of human and bacterial HSP epitopes may trigger the same activity and initiate the production of antibodies, primarily directed against pathogens and then, due to the mimicry, cross-reacting with the human HSP through TLR. In vitro experiments have shown that the chlamydial Hsp60, similar to that in humans, could induce IL-6 overproduction and the adhesion molecule overexpression on the endothelium, smooth muscle cells, and macrophages [15]. The additional proinflammatory and detrimental activity, observed in mice, was the result of the formation of the HSP-anti-HSP complexes within the endothelial and smooth muscle cells [16]. Contrarily, the low anti-Hsp60 and anti-Hsp70 levels in humans seem to act as negative prognostic factors, because they were connected with the increased risk of the acute coronary syndrome, whereas the highest values of these anti-HSP were observed in the healthy subjects $[10,11]$. Those discrepancies strongly suggest that the net effect of the HSP activity is the result of a balance and a negative biofeedback created between HSP and anti-HSP. Therefore, the increased HSP may trigger the production of the antibodies that, in turn, would create the HSP-anti-HSP complexes, thus eliminating the excessive circulating forms. The presence of such interactions was proved in the case of the Hsp60 - anti-Hsp60 and Hsp70 - antiHsp70 couples [10, 11].

\section{HSP in chronic kidney disease (CKD)}

$\mathrm{CKD}$, due to the complexity of the underlying disturbances and the variety of complications, seems an ideal example of the cell and organ responses to the long-lasting multifactorial stress conditions. The potential components of this "stress cocktail" are: the uremic toxins, mediators of inflammation, reactive oxygen species, apoptosis, infections, and, finally, the dialysis itself. Therefore, the discussion about the role of HSP in chronic kidney disease should be conducted in two parallel directions. The first one is connected with the process of chronic renal damage and the possible impact of HSP, either protective or deleterious, on its progression. Secondly, it is a question of defining the role of HSP in the possible therapeutic interventions, such as the modification in the biocompatibility of dialysis materials or the transplantation procedures, and the future perspectives in optimizing the renal replacement therapy and improving the patients' outcome.

\section{HSP in the progression of chronic kidney disease}

The in vitro investigation performed in the late 1990s has shown increased Hsp72 expression in human neuroblastoma cells treated with urea at the concentrations ranging from 40 to $200 \mathrm{mg} / \mathrm{dl}$. The HSP values rose after $30 \mathrm{~min}$, obtained their peak after $10 \mathrm{~h}$, and returned to zero after 48 $\mathrm{h}$ [17]. A similar experiment with creatinine at high concentrations $(0.5-14 \mathrm{mg} / \mathrm{dl})$ had no impact on the Hsp72 expression. This was the proof for the selective influence of uremic toxins on the stress response and a suggestion that the increase of Hsp72 expression may protect against apoptosis and adapt the cell to the noxious conditions. However, the short duration of the experiment (only hours) could not exactly mimic the conditions characteristic for chronic damage of the kidney. Therefore, the interpretation of the adaptive response in the course of CKD must be cautious. Similarly, the well-documented anti-apoptotic and protective role of Hsp27, Hsp72, and Hsp90 in the ischemia-reperfusion injury clearly shows the dynamic adaptive changes after kidney transplantation 
(discussed later), but gives no idea about what is happening during the long-time multifactorial CKD-related stress [18-20].

The only data concerning the impact of HSP on chronic kidney damage are those of Mao et al., who examined rats with obstructive nephropathy [21]. The selective activator of Hsp72, given orally, inhibited the proliferation and apoptosis in tubular cells, and diminished the accumulation of fibroblasts and collagen I in renal parenchyma, thus slowing the process of fibrosis.

On the other hand, Lin et al. [22] have shown the decreased expression of $\mathrm{Hsp} 27$ in the aortas of rats undergoing subtotal nephrectomy. This deficiency was most evident in the advanced atherosclerotic lesions, thus confirming the potentially cytoprotective role of this HSP and its impact on atherogenesis.

Contrarily, the investigation of mice with rapidly progressive glomerulonephritis has proven the detrimental impact of HSP on renal function. The Hsp60 injection provoked a dramatic worsening of kidney function, glomerular necrosis, and anuria [23]. There are no experimental data on the role of Hsp90 in chronic kidney disease.

Research on HSP in humans with CKD treated conservatively is limited to two studies. The first one [24] has described decreased Hsp72 expression in the peripheral blood monocytes from the adult patients, thus suggesting the exhaustion of the adaptive mechanisms, concordant with the aggravated apoptosis and the impaired immunity characteristic for CKD. The unchanged serum concentrations of Hsp70 in children with CKD [25] could speak in favor of the adaptive processes as being better preserved, but the concomitant decrease of anti-Hsp70, a negative prognostic factor in the cardiovascular complications, seems to confirm the increased risk for atherosclerosis in the course of CKD. The same study revealed diminished serum concentrations of Hsp60 and the increased values of anti-Hsp60. Although the low Hsp60 values in the light of previously mentioned studies seem profitable, it is difficult to regard them as so, if at the same time they are lower than in the control group. The probable explanation is the balance between HSP and anti-HSP that has been moved towards anti-Hsp60 with the consequence of the Hsp60 inhibition and decrease of its pool. Moreover, there is no doubt that the serum anti-Hsp60 acts negatively, accelerating the development of atherosclerosis. The elevated Hsp $90 \alpha$ concentrations in the same group may indicate an enhanced oxidative stress and inflammation, which are characteristic for CKD and trigger atherogenesis. The correlations between Hsp90 $\alpha$, anti-Hsp60, and sE-selectin, the marker of endothelial activation in children with CKD, also suggest a role of HSP in the development of atherosclerosis [25].

\section{HSP in the patients on dialysis}

Contrary to the scarce data concerning HSP in CKD patients on conservative treatment, much more has been done in the field of dialysis. The data have focused mainly on the role of HSP as the potential markers of the biocompatibility of materials that are used, although recent investigations seem to reveal a new trend towards pharmacologic interventions potentiating the protective abilities of HSP. However, the fact that patients on dialysis are at a greater risk of developing cardiovascular complications than those in a pre-dialysis state should also be taken into account while searching for the connections between HSP and dialysis modalities.

\section{HSP in hemodialysis (HD)}

The studies of HSP in hemodialysis have concentrated mainly on Hsp72. It has been shown that, in regard to the peripheral blood monocytes, the mRNA amount in adults on HD was significantly lower than in the controls [24]. When macrophages were analyzed, no such difference was observed in baseline Hsp72 expression, but response to induction by the high temperature (exposure to $47^{\circ} \mathrm{C}$ for 40 min) was impaired and accompanied by increased apoptosis [26]. Interestingly, the exposure of the urea-treated (150 $\mathrm{mg} / \mathrm{dl})$ macrophages, harvested from the healthy controls, to heat stress $\left(47^{\circ} \mathrm{C}\right.$ for $\left.40 \mathrm{~min}\right)$ resulted in a greater increase of Hsp72 expression than after the incubation with urea only. This could be the indirect proof of the previously mentioned results, when the high urea concentrations induced Hsp72 in human neuroblastoma cells [17]. On the other hand, it suggests that the stress response, although altered in CKD, is not entirely abolished. These results seem concordant with those of Crowe et al. who analyzed the role of HSP in skeletal muscle atrophy typical for patients on hemodialysis [27]. The analysis of muscle biopsies revealed increased content of Hsp27 and a tendency towards higher values of Hsp60 and Hsp70, probably being an adaptive response to the stress conditions evoked by dialysis.

The study performed in children and young adults revealed no difference in the Hsp70 and anti-Hsp70 serum concentrations between the subjects on hemodialysis and controls, thus questioning the negative impact of dialysis on the Hsp70 function in the pediatric population [28]. Although the Hsp60 values in the HD patients were decreased, they did not differ from those in the predialysis patients, which was similar to the situation observed in the case of Hsp70. The only exceptions were the levels of Hsp90 $\alpha$ and anti-Hsp60, significantly higher in the HD subjects than in both controls and the pre-dialysis 
patients. The case of $H \operatorname{sp} 90 \alpha$ can be explained by the oxidative stress and chronic inflammation undergoing aggravation due to dialysis. The increased anti-Hsp60 values point at the activation of the immune response, taking place in the course of HD. Taking into account the observed inverse correlation between Hsp60 and antiHsp60, the increased concentrations of the latter may also be responsible for the Hsp60 suppression.

The two studies, assessing the impact of a single dialysis session with the polysulfone membranes on HSP, have revealed an increase of Hsp72 expression [29], high Hsp60, as well as decreased anti-Hsp60 and anti-Hsp70 concentrations after HD [28]. The Hsp70 activation seems indicative of a reaction to the stressful conditions, such as the blood-dialyzer contact. The Hsp60 increase could also be the result of a protein release from the activated or damaged cells after their contact with the dialyzer membrane. Contrarily, there are many possible reasons for the decrease in the antibody levels. Indeed, the adsorption to the membrane surface, the creation of the HSP - anti-HSP complexes and the elimination with the dialysis solution have to be taken into account as probable explanations. However, the clinical interpretation based on a single study seems risky. While the decrease of the autoimmune activity may be profitable, the diminished concentrations of the antibodies would neglect the protective impact of anti-HSP on the cardiovascular system. On the other hand, the total cholesterol and the HDL-cholesterol levels predicted the anti-Hsp60 concentrations both before and after a single HD session, suggesting the relation of that antibody with the atherosclerosis development in this group of patients [28]. Therefore, the authoritarian defining of the role of Hsp60 and anti-Hsp60 is currently impossible, not only because of the HSP-antiHSP interactions but also due to their ambiguous function [30]. However, the above-mentioned results indicate the role of hemodialysis in aggravating the HSP disturbances, probably influencing atherogenesis and as is already seen in children with CKD on conservative treatment [25].

\section{HSP in peritoneal dialysis}

The investigations of HSP in peritoneal dialysis have mainly focused on the impact of the peritoneal fluid, as a stressor, on the function of the peritoneum. The induction of the Hsp72 expression has been confirmed in both mesothelial cells incubated with the dialysis fluid [31] and in macrophages from the dialysis effluent collected after a 4-h dwell [32]. In the meantime, the susceptibility of this protein to the fluid content has been proven, thus pointing to $\mathrm{Hsp} 72$ as a possible marker of the peritoneal fluid biocompatibility [32, 33]. Endemann et al. [34] went further and proved the role for HSP in the stabilization of the cytoskeleton of mesothelial cells exposed to the peritoneal dialysis solution (PDS). Indeed, the in vitro exposure of human mesothelial cells to PDS resulted in the overexpression and the shift of Hsp27 and Hsp72 from the noncytoskeletal to the cytoskeletal fraction within the cells. The incubation of the cytoskeletal protein, ezrin, with the recombinant Hsp72, stabilized this protein within the cytoskeletal fraction, whereas anti-Hsp72 caused its shift to the nonskeletal fraction. Moreover, the Hsp72 overexpression was predominant in the mesothelial cells detached after the exposure to PDS. Finally, the pretreatment of mesothelial cells with hyperthermia caused the upregulation of Hsp72 and reduced detachment after the contact with PDS.

The newest findings are these of Bender et al. [35] who successfully improved the status of mesothelial cells by the in vitro pharmacologic manipulation of the PDS content. In detail, the solution enriched in glutamine improved the viability of human mesothelial cells by inducing Hsp27 and Hsp72 expression. In the in vivo rat model, the same solution reduced the detachment of mesothelial cells and, what is more, decreased the amount of the protein that was lost with PDS.

The only study on extracellular HSP concerns children treated with automated peritoneal dialysis (APD) [25]. The serum concentrations of Hsp60 and anti-Hsp70 were diminished, whereas those of Hsp $90 \alpha$ and anti-Hsp60 were increased in comparison to the controls. When the APD children and the patients with pre-dialysis CKD were compared, the first ones had significantly higher Hsp90 $\alpha$ and anti-Hsp60 values, thus confirming the impact of dialysis on aggravating oxidative stress and autoimmune reactions. However, due to the fact that there were no such differences in the case of Hsp60 and anti-Hsp70, it is difficult to give an accurate opinion on the influence of APD on the quality of stress response in children.

An effort has also been made to answer the question as to whether there is any difference between hemodialysis and peritoneal dialysis, in regard to HSP. When the children treated with both modalities were compared, it appeared that the Hsp60 and Hsp90 $\alpha$ concentrations were similar in both groups [36]. Contrarily, the values of anti-Hsp60 in the HD population were significantly higher than those in the APDs, thus confirming the previously suggested negative impact of hemodialysis, in comparison to peritoneal dialysis, on the stress response in children. The antiHsp70 values, although higher in HD than in APD, remained unchanged versus the control group, so the interpretation of a negative impact of hemodialysis, based on this result, seems questionable. The possible explanation for the unchanged anti-Hsp70 values might be the inhibition by Hsp70, overexpressed in the course of a hemodialysis session [29]. 
The above-mentioned results have revealed the complexity of the HSP response to stressful conditions, showing that antiHSP might differentiate between the two dialysis modalities and become useful markers of biocompatibility. Although the HSP disturbances were more evident in the case of hemodialysis, this does not justify an explicitly negative opinion on that method, in regard to the impact on the stress response, in comparison to peritoneal dialysis. However, it may suggest that in children with end-stage renal disease, put in the majority of cases into peritoneal dialysis, such choice is less harmful than hemodialysis.

\section{HSP in kidney transplantation}

The transplanted kidney undergoes ischemia-reperfusion injury and HSP seem to play an essential role in that process. The investigation of rat renal cortex after ischemia has revealed the influence of overexpressed Hsp25, Hsp70, and Hsp90 on its repair by the relocation of the Na,K-ATPase from the apical to the basolateral membrane domain of the proximal tubule cells [18]. The results were confirmed by the fact that anti-HSP inhibited the translocation. Interestingly, the stabilizing power of HSP towards Na,K-ATPase was most efficient when all three of the HSP mentioned above acted together, suggesting that "the HSP cocktail" is more potent than any HSP alone. Surprisingly, another animal study by Fekete et al. has shown that, due to the elevated Hsp72 expression and the higher $\mathrm{Na}$, K-ATPase activity, female rats are more resistant to the ischemic changes in the kidney than are male rats [37].

The study in children after kidney transplantation has suggested that the urinary excretion of Hsp 72 is characteristic only for the early post-transplant period, whereas the patients with stable grafts do not have detectable levels of this HSP in the urine [38]. They have also proven that the urine Hsp72 is a good marker for loss of tubular cell integrity.

However, the essential concern for HSP in the organ transplantation is their potential role in preventing or delaying the process of rejection. The experimental data have shown that due to heat shock proteins, the cells preconditioned with sublethal stress become resistant and survive the subsequent, otherwise lethal, unfavorable stimulus. The natural consequence of such results was the question of whether the stimulation of HSP in the transplanted kidney would ameliorate the patient's outcome, and preserve the kidney function for a longer time or induce tolerance. Unfortunately, the results were ambiguous. The stress-induced Hsp70 overexpression improved the survival of the transplanted kidneys in both humans and rats [39, 40], whereas other experiments have shown that the Hsp70 expression has remained unchanged since the kidney engraftment until a few months after transplantation, irrespective of the graft function [41]. Contrarily, the
Hsp27 mRNA levels were significantly reduced in the rats with chronic allograft nephropathy, thus suggesting the potential profit coming from stimulating this HSP [42]. The observed translocation of Hsp27 from the medulla to the cortex seemed an adaptive response to the ischemic conditions. However, the possible consequences of the activation of such nonspecific agents as HSP might have been unpredictable and not necessarily profitable, like the increased immunogenicity [for review see 43].

Another perspective for therapeutic options probably lies in the blockade of TLR signaling, closely related to the HSP activity, which has recently been accused of contributing to chronic graft dysfunction [44]. The latter also suggests that the nearest future in the HSP research should concentrate on the interactions between these proteins, on the novel methods of their visualization in vivo $[45,46]$, as well as on the signaling pathways strictly connected with them.

\section{Conclusions}

Knowledge of the HSP engagement in chronic kidney disease is still increasing, although not all aspects of their role in this process are entirely understood. The intracellular forms of HSP, with Hsp70 above all, delay the progression of CKD by the anti-apoptotic activity and cytoprotection. The extracellular HSP in CKD show more variability in their activity, because they may actively protect against stress conditions (Hsp27, Hsp70), act detrimentally (Hsp60, anti-Hsp60), or be treated merely as the indicators of the accomplished cell damage $(H s p 90 \alpha)$. There is no doubt that dialysis aggravates the disturbances in stress response and the evidence points towards hemodialysis as a more deleterious technique. Preliminary data on the induction of protective HSP overexpression, resulting in the increased biocompatibility of dialysis fluids or better survival of the graft, give hope for the successful therapeutic interventions. Therefore, future investigation should concentrate on specifying the methods of HSP induction, thus improving the quality of renal replacement therapy in all its facets.

Open Access This article is distributed under the terms of the Creative Commons Attribution Noncommercial License which permits any noncommercial use, distribution, and reproduction in any medium, provided the original author(s) and source are credited.

\section{References}

1. Henderson B (2010) Integrating the cell stress response: a new view of molecular chaperones as immunological and physiological homeostatic regulators. Cell Biochem Funct 28:1-14 
2. Calderwood SK, Mambula SS, Gray PJ Jr (2007) Extracellular heat shock proteins in cell signaling and immunity. Ann NY Acad Sci 1113:28-39

3. Joly AL, Wettstein G, Mignot G, Ghiringhelli F, Garrido C (2010) Dual role of heat shock proteins as regulators of apoptosis and innate immunity. J Innate Immun 2:238-247

4. Pockley AG, Shepherd J, Corton JM (1998) Detection of heat shock protein 70 (Hsp70) and anti-Hsp70 antibodies in the serum of normal individuals. Immunol Invest 27:367-377

5. Pockley AG, Bulmer J, Hanks BM, Wright BH (1999) Identification of human heat shock protein 60 (Hsp60) and anti-Hsp60 antibodies in the peripheral circulation of normal individuals. Cell Stress Chaperones 4:29-35

6. Matzinger P (1994) Tolerance, danger, and the extended family. Annu Rev Immunol 12:991-1045

7. Srivastava $P$ (2002) Interaction of heat shock proteins with peptides and antigen presenting cells: chaperoning of the innate and adaptive immune responses. Annu Rev Immunol 20:395-425

8. Businaro R, Profumo E, Tagliani A, Buttari B, Leone S, D'Amati G, Ippoliti F, Leopizzi M, D'Arcangelo D, Capoano R, Fumagalli L, Salvati B, Rigano R (2009) Heat-shock protein 90: a novel autoantigen in human carotid atherosclerosis. Atherosclerosis 207:74-83

9. Zhu J, Quyyumi AA, Wu H, Csako G, Rott D, Zalles-Ganley A, Ogunmakinwa J, Halcox J, Epstein SE (2003) Increased serum levels of heat shock protein 70 are associated with low risk of coronary artery disease. Arterioscler Thromb Vasc Biol 23:1055-1059

10. Dulin E, Garcia-Barreno P, Guisasola MC (2010) Extracellular heat shock protein 70 (HSPA1A) and classical vascular risk factors in a general population. Cell Stress Chaperones 15:929-937

11. Zhang $\mathrm{X}, \mathrm{Xu} \mathrm{Z}$, Zhou L, Chen Y, He M, Cheng L, Hu FB, Tanguay RM, Wu T (2010) Plasma levels of Hsp70 and antiHsp70 antibody predict risk of acute coronary syndrome. Cell Stress Chaperones 15:675-686

12. Schmitt E, Gehrmann M, Brunet M, Multhoff G, Garrido C (2007) Intracellular and extracellular functions of heat shock proteins: repercussions in cancer therapy. J Leukoc Biol 81:15-27

13. Lamb DJ, El-Sankary W, Ferns GA (2002) Molecular mimicry in atherosclerosis: a role for heat shock proteins in immunisation. Atherosclerosis 167:177-185

14. Perschinka H, Mayr M, Millonig G, Mayerl Ch, Van der Zee R, Morrison SG, Morrison RP, Xu Q, Wick G (2003) Cross-reactive B-cell epitopes of microbial and human heat shock protein $60 / 65$ in atherosclerosis. Arterioscler Thromb Vasc Biol 23:1060-1065

15. Kol A, Bourcier T, Lichtman AH, Libby P (1999) Chlamydial and human heat shock protein $60 \mathrm{~s}$ activate human vascular endothelium, smooth muscle cells, and macrophages. J Clin Invest 103:571-577

16. Foteinos G, Afzal AR, Mandal K, Jahangiri M, Xu Q (2005) Antiheat shock protein 60 autoantibodies induce atherosclerosis in apolipoprotein E-deficient mice via endothelial damage. Circulation 112:1206-1213

17. Maddock AL, Westenfelder C (1996) Urea induces the heat shock response in human neuroblastoma cells. J Am Soc Nephrol 7:275282

18. Bidmon B, Endemann M, Muller T, Arbeiter K, Herkner K, Aufricht C (2002) HSP-25 and HSP-90 stabilize Na, K-ATPase in cytoskeletal fractions of ischemic rat renal cortex. Kidney Int 62:1620-1627

19. Kabakov AE, Budagova KR, Bryantsew AL, Latchman DS (2003) Heat shock protein 70 or heat shock protein 27 overexpressed in human endothelial cells during posthypoxic reoxygenation can protect form delayed apoptosis. Cell Stress Chaperones 8:335-347

20. Van Why SK, Siegel NJ (1998) Heat shock proteins in renal injury and recovery. Curr Opin Nephrol Hypertens 7:407-412
21. Mao H, Li Z, Zhou Y, Li Z, Zhuang S, An X, Zhang B, Chen W, Nie J, Wang Z, Borkan SC, Wang Y, Yu X (2008) HSP72 attenuates renal tubular cell apoptosis and interstitial fibrosis in obstructive nephropathy. Am J Physiol Renal Physiol 295:F202-F214

22. Lin YP, Hsu ME, Chiou YY, Hsu HY, Tsai HC, Peng YJ, Lu CY, Pan CY, Yu WC, Chen CH, Chi CW, Lin CH (2010) Comparative proteomic analysis of rat aorta in a subtotal nephrectomy model. Proteomics 10:2429-2443

23. Lang A, Benke D, Eitner F, Engel D, Ehrlich S, Breloer M, HamiltonWilliams E, Specht S, Hoerauf A, Floege J, von Bonin A, Kurts C (2005) Heat shock protein 60 is released in immune-mediated glomerulonephritis and aggravates disease: in vivo evidence for an immunologic danger signal. J Am Soc Nephrol 16:383-391

24. Marzec Ł, Zdrojewski Z, Liberek T, Bryl E, Chmielewski M, Witkowski JM, Rutkowski B (2009) Expression of Hsp72 protein in chronic kidney disease patients. Scand J Urol Nephrol 43:400-408

25. Musiał K, Szprynger K, Szczepańska M, Zwolińska D (2010) The heat shock protein profile in children with chronic kidney disease. Perit Dial Int 30:227-232

26. Reuter S, Bangen P, Edemir B, Hollebrand U, Pavenstaedt H, Heidenreich S, Lang D (2009) The HSP72 stress response of monocytes from patients on haemodialysis is impaired. Nephrol Dial Transplant 24:2838-2846

27. Crowe AV, McArdle A, McArdle F, Patwell DM, Bell GM, Kemp GJ, Bone M, Griffiths RD, Jackson MJ (2007) Markers of oxidative stress in the skeletal muscle of patients on hemodialysis. Nephrol Dial Transplant 22:1177-1183

28. Musiał K, Szprynger K, Szczepańska M, Zwolińska D (2009) Heat shock proteins in children and young adults on chronic hemodialysis. Pediatr Nephrol 24:2029-2034

29. Raj DS, Boivin MA, Dominic EA, Boyd A, Roy PK, Rihani T, Tzamaloukas AH, Shah VO, Moseley P (2007) Haemodialysis induces mitochondrial dysfunction and apoptosis. Eur J Clin Invest 37:971-977

30. Alard JE, Dueymes M, Youinou P, Jamin C (2007) Modulation of endothelial cell damages by anti-Hsp60 autoantibodies in systemic autoimmune diseases. Autoimmunity Rev 6:438-443

31. Arbeiter K, Bidmon B, Endenmann M, Ruffingshofer D, Mueller $\mathrm{T}$, Regele H, Eickelberg O, Aufricht C (2003) Induction of mesothelial HSP-72 upon in vivo exposure to peritoneal dialysis fluid. Perit Dial Int 23:499-501

32. Marzec Ł, Liberek T, Chmielewski M, Bryl E, Witkowski JM, Liberek K, Zdrojewski Z, Rutkowski B (2007) Expression of heat shock protein 72 in peritoneal leukocytes is induced by peritoneal dialysis. Perit Dial Int 27:288-295

33. Bender TO, Witowski J, Aufricht Ch, Endemann M, Frei U, Passlick-Deetjen J, Jorres A (2008) Biocompatibility of a bicarbonate-buffered amino-acid-based solution for peritoneal dialysis. Pediatr Nephrol 23:1537-1541

34. Endemann M, Bergmeister H, Bidmon B, Boehm M, Csaicsich D, Malaga-Dieguez L, Arbeiter K, Regele H, Herkner K, Aufricht C (2007) Evidence for HSP-mediated cytoskeletal stabilization in mesothelial cells during acute experimental peritoneal dialysis. Am J Physiol Renal Physiol 292:F47-F56

35. Bender TO, Bohm M, Kratochwill K, Lederhuber H, Endemenn M, Bidmon B, Aufricht C (2010) Hsp-mediated cytoprotection of mesothelial cells in experimental acute peritoneal dialysis. Perit Dial Int 30:294-299

36. Musiał K, Szczepańska M, Szprynger K, Zwolińska D (2009) The impact of dialysis modality on serum heat shock proteins in children and young adults with chronic kidney disease. Kidney Blood Press Res 32:366-372

37. Fekete A, Vannay A, Ver A, Rusai K, Muller V, Reusz G, Tulassay T, Szabo AJ (2006) Sex differences in heat shock protein 72 expression and localization in rats following renal ischemiareperfusion injury. Am J Physiol Renal Physiol 291:F806-F811 
38. Mueller T, Bidmon B, Pichler P, Arbeiter K, Ruffingshofer D, van Why SK, Aufricht C (2003) Urinary heat shock protein-72 excretion in clinical and experimental renal ischemia. Pediatr Nephrol 18:97-99

39. Trieb K, Dirnhofer S, Krumbock N, Blahovec H, Sgonc R, Margreiter R, Feichtinger H (2001) Heat shock protein expression in the transplanted human kidney. Transplant Int 14:281-286

40. Redaelli CA, Wagner M, Kulli C, Tian YH, Kubulus D, Mazzucchelli L, Wagner AC, Schilling MK (2001) Hyperthermia-induced HSP expression correlates with improved rat renal isograft viability and survival in kidneys harvested from non-heart-beating donors. Transplant Int 14:351-360

41. Mueller T, Regele H, Posch M, Marszalek M, Schwarz C, Pichlhoefer B, Arbeiter K, Aufricht C (2004) Hsp72 expression in pre-transplant donor kidney biopsies and post-transplant outcome. Transplantation 78:292-295
42. Djamali A, Reese S, Oberlet T, Hullett D, Becker B (2005) Heat shock protein 27 in chronic allograft nephropathy: a local stress response. Transplantation 79:1645-1657

43. Pockley AG (2001) Heat shock proteins, anti-heat shock protein reactivity and allograft rejection. Transplantation 71:1503-1507

44. Wang S, Schmaderer C, Kiss E, Schmidt C, Bonrouhi M, Porubsky S, Gretz N, Schaefer L, Kirschning CJ, Popovic ZV, Grone HJ (2010) Recipient Toll-like receptors contribute to chronic graft dysfunction by both MyD88- and TRIF-dependent signaling. Dis Model Mech 3:92-103

45. Wick MC, Kremser Ch, Frischauf S, Wick G (2008) In vivo molecular imaging of vascular stress. Cell Stress Chaperones $13: 263-273$

46. Wick MC, Mayerl Ch, Backovic A, van der Zee R, Jaschke W, Dietrich H, Wick G (2008) In vivo imaging of the effect of LPS on arterial endothelial cells: molecular imaging of heat shock protein 60 expression. Cell Stress Chaperones 13:275-285 